\title{
Guidelines for choice of selective serotonin reuptake inhibitor in depressive illness
}

\author{
Ian M. Anderson \& J. Guy Edwards
}

The selective serotonin reuptake inhibitors (SSRIs) are the most extensively studied of the newer antidepressants and are increasingly being used as firstline treatment for depression (Anderson et al, 2000). In this article we concentrate on issues that need to be taken into account when selecting one of the five SSRIs marketed in the UK (citalopram, fluoxetine, fluvoxamine, paroxetine and sertraline) for individual patients. We have concentrated on treatment of depression and have not reviewed their use in anxiety disorders because comparative data are lacking.

\section{Quality of evidence determining choice}

Randomised controlled trials (RCTs) are the gold standard for quality of evidence but they have several limitations. They do not provide direct evidence on effectiveness (how well a treatment works in practice) as opposed to efficacy (how well it works in ideal conditions). Most RCTs are relatively short term, whereas longer-term data are required for treatment recommendations. The use of strict inclusion and exclusion criteria means that patients in trials are unlikely to match those encountered in clinical practice. RCTs are expensive and most contain relatively few patients, so that less common treatment-emergent events are unlikely to be detected. In the fiercely competitive area of antidepressant treatment, most
RCTs are a marketing tool designed to detect (or refute) areas in which one drug may outperform another and their results are presented with a particular emphasis.

A systematic review and meta-analysis allows some of the potential biases to be addressed so that individual studies are not given undue weight. Furthermore, to complement RCTs it is important to turn to other types of data, although they all have their limitations. Naturalistic studies encompass a variety of designs involving the follow-up of patients treated routinely in clinical practice. In these studies treatment is open, so that biases in initial selection of antidepressant and subsequent management are likely to influence the outcome. All methods of population monitoring, from prescription databases to adverse event surveillance, similarly have these general potential confounds as well as their individual methodological assumptions. Case reports may draw attention to potential issues but can never in themselves provide a basis for comparison between drugs. Finally, extrapolation from pharmacological properties may predict certain events or outcomes but their clinical importance has to be tested empirically.

\section{Pharmacology}

Selective serotonin reuptake inhibitors differ in their potency and selectivity in inhibiting serotonin

Ian Anderson is a senior lecturer and honorary consultant psychiatrist at Manchester Royal Infirmary (University of Manchester, Neuroscience and Psychiatry Unit, Room G809, Stopford Building, Oxford Road, Manchester M13 9PT). He is Secretary of the British Association for Psychopharmacology. His research interests are in the psychopharmacology of affective disorders and in evidence-based psychiatry. Guy Edwards is a consultant psychiatrist at the Chalybeate Hospital, Southampton, an honorary clinical senior lecturer in the Department of Psychiatry at Royal South Hants Hospital and Visiting Professor, Khon Kaen University, Thailand. He was the founding Editor-in-Chief of Human Psychopharmacology: Clinical and Experimental. 
Table 1 Clinically important interactions between selective serotonin reuptake inhibitors and cytochrome P450 isoenzymes (CYPs)

\begin{tabular}{|c|c|c|c|c|c|}
\hline & Citalopram & Fluoxetine & Fluvoxamine & Paroxetine & Sertraline \\
\hline CYP inhibited & None & $\begin{array}{l}\text { 2C19; 2D6 } \\
3 \mathrm{~A} 3 / 4\end{array}$ & $\begin{array}{l}1 \mathrm{~A} 2 ; 2 \mathrm{C} 19 \\
3 \mathrm{~A} 3 / 4\end{array}$ & 2D6 & 2D6 (weak) \\
\hline $\begin{array}{l}\text { Selected drugs with } \\
\text { potential for } \\
\text { interaction at level } \\
\text { of hepatic metabolism }{ }^{2}\end{array}$ & None & $\begin{array}{l}\text { Anti-arrythmics; } \\
\text { antipsychotics; } \\
\text { opiates; } \\
\text { phenytoin } \\
\text { TCAs }\end{array}$ & $\begin{array}{l}\text { Antipsychotics; } \\
\text { clozapine; } \\
\text { diazepam; } \\
\text { TCAs }\end{array}$ & $\begin{array}{l}\text { Anti-arrythmics; } \\
\text { antipsychotics; } \\
\text { opiates; } \\
\text { TCAs; } \\
\text { theophylline }\end{array}$ & $\begin{array}{l}\text { Probably } \\
\text { insignificant }\end{array}$ \\
\hline
\end{tabular}

TCA, tricyclic antidepressant.

1. Mainly via metabolite norfluoxetine (seproxetine).

2. List not exhaustive and does not include interactions at other sites; British National Formulary should be consulted.

reuptake and there may be important effects on other transporters and receptors, indicating that they are not as selective as their manufacturers suggest. It has been proposed, for example, that fluoxetine's effects on $5-\mathrm{HT}_{2 \mathrm{C}}$ receptors may underlie a propensity to cause agitation; paroxetine's affinity for muscarinic receptors causes its increased tendency to produce discontinuation effects; sertraline's affinity for the dopamine transporter results in a greater efficacy in severe depression; and sertraline's and fluvoxamine's affinity for sigma ${ }_{1}$ opioid receptors is responsible for their efficacy in psychotic depression (Goodnick \& Goldstein, 1998a). However, at our current state of knowledge pharmacodynamic differences between the SSRIs are of minor practical importance in determining choice. They are used to try to explain observed or suggested clinical effects that have varying degrees of evidential support.

Pharmacokinetic differences between SSRIs are more important. Fluoxetine differs in having a much longer elimination half-life (1-3 days, seproxetine (norfluoxetine - its active metabolite) 7-15 days) than that of its competitors, which range from about 0.5 to 1.5 days. This means that it takes longer to reach steady state (and possibly effective) plasma concentrations and that a substantially longer washout period is required if drugs that interact with fluoxetine are to be prescribed. Another important area in which SSRIs differ is in their propensity to inhibit hepatic cytochrome P450 (CYP) isoenzymes, which are responsible for the metabolism of many drugs (see Table 1).

\section{Efficacy and effectiveness}

The SSRIs are all licensed for use in depressive disorders but differ in their licensed indications for other disorders (which differ between countries). Although the choice of SSRI based on product licence may be appropriate in certain circumstances, it is not based on sound evidence. Comparative studies of SSRIs are rare in disorders apart from depression, making informed comparisons difficult, if not impossible in these conditions. The UK licensed indications are summarised in Table 2, but they will not be considered further in this article. Instead, we will concentrate on the evidence for choosing specific SSRIs in depression - the most common indication for prescribing them (Lawrenson et al, 2000).

Table 2 Licensed indications for serotonin selective reuptake inhibitors in the UK as of February 2001

\begin{tabular}{|c|c|c|c|c|c|}
\hline Indication & Citalopram & Fluoxetine & Fluvoxamine & Paroxetine & Sertraline \\
\hline Depressive illness & $\checkmark$ & $\checkmark$ & $\checkmark$ & $\checkmark$ & $\checkmark$ \\
\hline Panic disorder & $\checkmark$ & & & $\checkmark$ & \\
\hline Obsessive-compulsive disorder & & $\checkmark$ & $\checkmark$ & $\checkmark$ & $\checkmark$ \\
\hline Social phobia & & & & $\checkmark$ & \\
\hline Premenstrual dysphoric disorder & & $\checkmark$ & & & \\
\hline Bulimia nervosa & & $\checkmark$ & & & \\
\hline Post-traumatic stress disorder & & & & $\checkmark$ & \\
\hline
\end{tabular}




\section{Efficacy in depressive disorder}

The comparative efficacy of SSRIs can only be confidently assessed from head-to-head RCTs. We systematically reviewed 21 such trials in depression (Edwards \& Anderson, 1999), 16 of which involved comparisons with fluoxetine. As might be expected, there were apparent differences between SSRIs reported in some of the trials (many of which had marketing in mind) - particularly those that presented post hoc or subgroup analyses. To minimise bias and overemphasis in the results from individual studies, we carried out a metaanalysis of the 20 studies, from which quantitative data were available. We compared each individual SSRI with the comparator SSRIs. Variance-weighted pooling of the relative effect size for each study showed no consistent differences in efficacy at endpoint in what were mostly short-term trials (typically of 6-8 weeks duration). However, the reduction in Hamilton Rating Scale for Depression (HRSD; Hamilton, 1960) scores was significantly less on fluoxetine than other SSRIs after 2-3 weeks of treatment, supporting the findings from some individual studies that fluoxetine may have a slower onset of action. The effect size was small and of uncertain clinical significance. The result may be explained by the pharmacokinetic difference between SSRIs.

Further comparative RCTs have been reported since this meta-analysis was carried out; none of these studies appears to be incompatible with the efficacy findings from our earlier meta-analysis.

\section{Outcome in naturalistic studies}

In general, studies looking at the naturalistic use of antidepressants have focused on the process or economics of treatment rather than outcome. Exceptions are two small retrospective studies of outcome in primary care. In the first of these, a study of 194 patients receiving one of the five SSRIs, no difference in outcome between the drugs was found (Arias et al, 1998). The second study involved 160 patients receiving fluoxetine, fluvoxamine, paroxetine or sertraline (Hylan et al, 1999). Patients who did not switch antidepressant or increase the dose (those on stable therapy) were 1.6 times more likely to respond than those who had a change in their regimen. Consistent with other descriptive studies, patients started on fluoxetine were more likely to have stable therapy than those on other SSRIs. Controlling for factors influencing outcome, patients on sertraline were about half as likely to respond to treatment as those on fluoxetine. There was no difference between the other SSRIs. These studies are, however, likely to be confounded by prescribing practice, for example, which SSRI is used first-line (likely to be associated with stable treatment and better outcome) and which second- or third-line (likely to be associated with relative treatment resistance).

\section{Other aspects of outcome}

Much has been made of the possibility of different SSRIs having different profiles of action according to patient characteristics, such as differential effects on anxious depression, melancholia, psychotic depression or depression with cognitive dysfunction (Goodnick \& Goldstein, 1998b). In addition, some studies have looked at outcome from a wider perspective than changes in scores on rating scales, for example quality of life measures. Such studies have suggested possible advantages of one SSRI over another in social functioning or performance at work (Goodnick \& Goldstein, 1998b; Claxton et al, 1999; Edwards \& Anderson, 1999). Although many of these findings come from RCTs, our view is that it is not possible at present to distinguish true differences from chance findings, selective reporting and commercial emphasis for marketing purposes. It is difficult to base recommendations for choice of an SSRI on either the clinical presentation (with the possible exception of fluoxetine for depression with agitation) or quality of life measures.

\section{Tolerability}

\section{Randomised controlled trials}

Nineteen of the 21 studies in our meta-analysis reported the total number of patients discontinuing treatment and those who were said to have discontinued because of adverse events (Edwards \& Anderson, 1999). Approximately 3000 patients were included in the trials, with $26 \%$ stopping treatment early $-11 \%$ allegedly owing to adverse events. The most consistent finding was a greater risk of dropping out of treatment while on fluvoxamine compared with the other SSRIs. A similar result was found for discontinuation thought by the trialists to be due to adverse effects. The respective numbers needed to harm were 14 and 16 . It seems likely that this difference between fluvoxamine and the other SSRIs is in part dose-related, as there was a significant correlation between the drop-out rate owing to adverse effects and the dose of fluvoxamine. At a dose of $100 \mathrm{mg}$ the drop-out rate was comparable to the other SSRIs. There were no 
other differences in overall drop-outs between SSRIs.

In our analysis of nine studies comparing sertraline with other SSRIs, there were fewer dropouts attributed to adverse reactions during treatment with sertraline, with a number needed to treat of 19 . However, four further studies involving sertraline have since been reported and a preliminary analysis of the data suggests that there is no significant advantage to sertaline in discontinuation rates attributed to adverse effects (unpublished data: details available from I.M.A. upon request).

There are limits to what can be determined from RCTs in terms of specific types of side-effect because of the selective reporting of results. In our review, nausea was reported in 17 studies; it occurred in $21 \%$ of patients, with no difference between SSRIs. The reporting of other side-effects was much more selective. The incidence of agitation was reported in only six out of 16 trials of fluoxetine and nervousness/anxiety in only 10 . Although slightly more patients had these side-effects while on fluoxetine than on its comparators (a difference that was significant only for agitation), it is difficult to exclude a reporting bias. Other effects noted more frequently with fluoxetine in more than one study were dermatological reactions/rashes (three studies) and weight loss (four studies, two of which were comparisons with paroxetine). A three-way RCT of fluoxetine $v$. sertraline $v$. paroxetine in 284 patients (Fava et al, 1998) reported significant weight gain on paroxetine over 6 months (with a quarter of the patients experiencing more than a $6 \%$ increase in weight). This did not occur in the case of the other two SSRIs. This difference between SSRIs may be of significance for individual patients but is of uncertain overall importance.

There are suggestive data from the Committee on Safety of Medicines (CSM) that treatment with paroxetine is more likely to be followed by discontinuation reactions than treatment with other SSRIs. This finding gains support from two studies in which patients on fluoxetine, paroxetine or sertraline had treatment interrupted by double-blind placebo substitution for 4-8 days. The first involved 242 patients maintained on one of the three SSRIs (Rosenbaum et al, 1998). Abrupt placebo substitution resulted in significant increases in discontinuation symptoms in the paroxetine and sertraline groups, but not in the fluoxetine group. In a subsequent RCT, 284 patients had placebo substitution after 22 weeks of treatment. Similar results were obtained, with the incidence of discontinuation symptoms in the sertraline group being intermediate between that of paroxetine and fluoxetine, although not significantly greater than the latter (Michelson et al, 2000).

\section{Naturalistic and surveillance data}

Committee on Safety of Medicines

Data from the CSM are useful for identifying adverse reactions to newly marketed drugs, but they do not allow the relative frequency of reactions to different drugs to be assessed with confidence. In the first 2 years of marketing, the number of reactions reported per 1000 prescriptions were greatest for fluvoxamine (25), followed by paroxetine (12), fluoxetine (7), sertraline (7) and citalopram (3) (Edwards \& Anderson, 1999). Apart from paroxetine, this order roughly parallels the order in which the drugs were launched on to the market in the UK, which could partly account for the differences.

Considering data from the drugs' introduction into practice up to September 1998, the most common adverse reaction reported for all SSRIs was nausea. The next four most common reactions in decreasing order of frequency were: for citalopram, headache, tremor, dizziness and sweating; for fluoxetine, headache, urticaria, rash (unspecified) and agitation; for fluvoxamine, vomiting, diarrhoea, tremor and dizziness; for paroxetine, discontinuation reaction, dizziness, tremor and headache; and for sertraline, diarrhoea, headache, dizziness and tremor. These data do not allow detailed direct comparisons between SSRIs to be made, but are consistent with those from other sources. They suggest that, compared with other SSRIs, fluoxetine may be more likely to cause dermatological reactions and agitation, fluvoxamine more severe gastrointestinal side-effects and paroxetine more severe discontinuation reactions.

\section{Prescription event monitoring}

Prescription event monitoring (PEM) studies have revealed that, of events considered to be of sufficient importance to be recorded in general practitioner records: (a) nausea and vomiting were reported more often during treatment with fluvoxamine than fluoxetine, paroxetine and sertraline; (b) drowsiness and tremor more often with fluvoxamine and paroxetine than fluoxetine and sertaline; and (c) sweating, male sexual dysfunction and withdrawal reactions more often with paroxetine than fluoxetine, fluvoxamine and sertraline. Overall, more events were recorded during treatment with fluvoxamine than the other three SSRIs (Edwards et al, 1997; Mackay et al,1997, 1999). A PEM study of citalopram had not been carried out when these comparisons were published. 


\section{Specific adverse reactions}

\section{Sexual dysfunction}

There are suggestions from individual trials and from observational studies that sexual side-effects are more liable to occur during treatment with some SSRIs than others. For instance, it was reported that sexual dysfunction occurred more frequently during treatment with sertraline than fluvoxamine (Nemeroff et al, 1995), while one PEM publication suggested that impotence and ejaculatory failure occurred more often during treatment with paroxetine than fluoxetine, fluvoxamine and sertraline (Mackay et $a l, 1997)$. A prospective descriptive study of 344 male and female out-patients found that paroxetine was associated with significantly more impotence and delayed orgasm than fluoxetine, fluvoxamine and sertraline. Fluvoxamine was associated with the lowest prevalence of sexual dysfunction, although this was not significantly different from fluoxetine or sertraline (Montejo-Gonzalez et al, 1997).

Our meta-analysis failed to reveal any significant difference between the individual SSRIs in their tendency to cause sexual side-effects. It is, however, difficult to interpret these findings because of incomplete reporting and different definitions of the types of sexual dysfunction described.

Of interest is an RCT comparing the effects of 6 weeks' treatment with fluoxetine, fluvoxamine, paroxetine, sertraline and placebo on delaying the time to ejaculation in men with life-time rapid ejaculation (intravaginal ejaculation delay of 1 minute or less). Fluvoxamine was the only SSRI not to differ significantly from placebo, with paroxetine delaying ejaculation most strongly. This effect of paroxetine was replicated in a group of men with rapid and less-rapid (intravaginal ejaculation delay of greater than 1 minute) ejaculation (Waldinger $e t$ al, 1998).

From the limited data there is a fairly consistent picture that, of the SSRIs, paroxetine may be the most likely to cause sexual dysfunction and fluvoxamine the least likely to cause this problem.

\section{Suicide and aggression}

Since the first case reports of suicidal and aggressive thoughts and behaviour allegedly provoked by fluoxetine (Teicher et al, 1990), there has been controversy about whether or not SSRIs, in particular fluoxetine, may cause some patients to become suicidal and/or abnormally aggressive. There are relatively few data on the rates of occurrence of these events, but PEM studies have revealed low rates, with little or no difference in incidence during the first 2 months after the start of treatment between fluoxetine, fluvoxamine and paroxetine (Edwards et al, 1997; Mackay et al, 1997).

\section{Drug interactions}

The British National Formulary (British Medical Association \& Royal Pharmaceutical Society, 2000) warns of higher numbers of interactions with fluoxetine and fluvoxamine than the other SSRIs. It might be considered that the apparently higher risk with these older SSRIs could be owing to selective reporting and the avoidance of concomitant medication previously shown to be hazardous when prescribed with the antidepressants. On the other hand, in vitro studies suggest that citalopram and sertraline should be the least likely to cause interactions because of their lower inhibition of cytochrome P450 enzymes (Table 1).

In practice the potential for serious interactions demands that clinicians should be aware of all substances taken by their patients (including illicit drugs and over-the-counter medication) and, when appropriate, choose an SSRI with the lowest potential for interactions.

\section{Safety in overdose}

The SSRIs are safer in overdose than the older tricyclic antidepressants (Henry et al, 1995), but six deaths following overdoses of citalopram raised the possibility that this drug could be more intrinsically toxic in overdose than the other SSRIs (Öström et al, 1996). However, no fatalities occurred among 44 patients who took overdoses of citalopram alone in quantities ranging from 70 to $3000 \mathrm{mg}$ (Personne $e t$ al, 1997). Widened QRS complexes in the electrocardiogram (ECG) were observed and/or convulsions occurred in about a third of patients who had taken $600-1800 \mathrm{mg}(30-90 \times 20 \mathrm{mg}$ tablets) and in all of those who had taken more than $1900 \mathrm{mg}$ ( $95 \times 20 \mathrm{mg}$ tablets). While there is uncertainty as to the cause of death in the citalopram fatalities, overdoses of the other SSRIs have not been associated with convulsions or ECG abnormalites (Dechant \& Clissold, 1991; Henry, 1991; Borys et al, 1992; Klein-Schwartz \& Anderson, 1996).

The six deaths reported by Öström et al (1996) have to be balanced against the extremely small number of successful suicides associated with citalopram 
overdose (Isacsson \& Bergman, 1996). In most lethal cases other anxiolytics (sedatives) were taken with citalopram and the quantity of citalopram taken in one of the suicides reported by Öström et al (1996) was similar to that in the only other well-documented death owing to an overdose of an SSRI taken alone - fluoxetine (Glassman, 1997).

The deaths owing to overdose of citalopram may well have been chance findings, but given the adverse events additionally reported by Personne et al (1997), clinicians might nevertheless wish to err on the side of safety and not choose this SSRI as first-choice treatment in patients particularly prone to self-poisoning.

\section{Safety in pregnancy and breast-feeding}

\section{Pregnancy}

Formal testing of drugs and inclusion in clinical trials during pregnancy and breast-feeding is unethical, yet the issue of safety is crucial in practice. After marketing there are invariably case reports of teratogenicity and other harm, but it is usually difficult to establish a cause-and-effect relationship between the drug and the abnormality. To help in the assessment of risk, prospective surveys, cohort-controlled studies and case-controlled studies have been carried out.

A PEM study reported the outcomes of pregnancy in 187 women treated with fluoxetine, fluvoxamine, paroxetine and sertraline. There was not a higher than expected incidence of spontaneous, missed or legal abortions, ectopic pregnancies, intra-uterine deaths or congenital abnormalities (Wilton et al, 1998). Goldstein \& Sundell (1999) reviewed five prospective surveys and four published cohortcontrolled studies of women who received SSRIs during pregnancy. The prospective survey involved more than 1000 women who had been treated with fluoxetine during the first trimester. There was not an increased risk of spontaneous abortion or major malformation. Nearly 300 other women had received fluvoxamine, paroxetine or sertraline - again with no apparent increased risk. In the cohort studies the rates of premature birth and postnatal complications following intra-uterine exposure to the SSRIs were not significantly different from those in the control groups and birth weights were similar. Pre-school children exposed to fluoxetine during pregnancy showed no significant difference from controls in global IQ, language or behaviour.

While these results provide grounds for cautious optimism, the numbers are relatively small and observational designs cannot formally establish safety. They do not, therefore, override the principle of avoiding drugs during pregnancy, particularly the first trimester, unless the balance of risks strongly favours treatment. If antidepressants are unquestionably indicated, the SSRI on which most data are available is fluoxetine.

\section{Breast-feeding}

SSRIs, like most drugs, are excreted in breast milk and there have been isolated case reports of suspected adverse reactions in breast-fed infants (e.g. crying, irritability and colic with fluoxetine). Limited data on mothers and babies are available, with little evidence of adverse consequences in the very small numbers reported - fluoxetine 30, fluvoxamine 2, paroxetine 2 and sertraline 26, with no data on citalopram (Yoshida et al, 1999).

The use of antidepressants in lactating mothers requires a balancing of risks that are impossible to quantify on available evidence. In view of the unknown effects of such drugs on the developing infant, a conservative view would be to prescribe only when there are unequivocal indications. The SSRIs on which most data are available are fluoxetine and sertraline, with fluoxetine having the potential disadvantage of a more prolonged effect should an adverse reaction occur.

\section{Other considerations}

\section{Dose escalation and switching}

In naturalistic studies in primary care, it has been reported that a better outcome is associated with a 'stable' pattern of antidepressant prescribing, that is, continuing on the first chosen antidepressant at the dose initially prescribed (Hylan et al, 1999; Claxton et al, 2000). This is not surprising because increasing the dose or switching antidepressants are strategies employed when patients do not respond to treatment. There are a number of studies in general practice showing that patients treated with fluoxetine are more likely to have a stable pattern compared with other SSRIs. However, results vary. In some studies there is a more stable pattern with fluoxetine than paroxetine but not sertraline, whereas in others there is more stability with fluoxetine than sertraline but not paroxetine (Gregor et al, 1994; Hylan et al, 1999; Russell et al, 1999; Lawrenson et al, 2000).

In contrast, a study in secondary care in Austria comparing citalopram, fluoxetine and paroxetine 
found no evidence of differential dose titration (Hylan et al, 1998). These naturalistic data are, however, crucially dependent on patterns of SSRI use, as antidepressants used as second- or thirdline treatment are more likely to be given to treatment-resistant patients. It is possible that the findings for fluoxetine in primary care simply reflect its more frequent use as first-line treatment.

There have been anecdotal or uncontrolled reports of an apparent favourable response to changing treatment from one SSRI to another. However, one cannot exclude the possibility that such responses are owing to chance, the continuing effect of drugs with a similar pharmacological action, or the psychological effect of change or the passage of time.

\section{Pharmacoeconomics}

The pharmacoeconomics of prescribing antidepressants are complex and lack consistent accepted methodology. Financial considerations and costs differ in different health care systems and their importance may depend on which budget is being considered. Conflicting results are often obtained (unsurprisingly favouring the study sponsor's drug), making it difficult to draw firm conclusions. For example, an RCT comparing sertraline and fluoxetine in primary care over 6 months in France found lower costs associated with sertraline (Boyer et al, 1998), as did a retrospective database analysis in the USA (Russell et al, 1999). In contrast, naturalistic studies in health maintenance organisations in the USA found that sertraline was associated with higher costs and greater health service utilisation than fluoxetine (Sclar et al, 1995, 1999).

\section{Guidelines for choice of SSRIs}

Difference between SSRIs are summarised in Table 3 , together with the type of evidence in support of the differences. In applying this comparison as a guide to choice (see Table 4), it is important to put in perspective what may be relatively small differences for most patients. The choice of antidepressant should be matched to individual patients' needs as far as possible (Anderson et al, 2000). This depends on the efficacy and tolerability of previous treatment with an SSRI; whether a particular side-effect such as sedation is required; and the risks of interactions and lethality in overdose. It is also good practice to limit the amount of drug available, especially if there is a serious risk of overdose.

In comparing SSRIs, limitations regarding the available evidence must be recognised. In general, there are fewer comparative data for citalopram and fluvoxamine than the other SSRIs, possibly because fluvoxamine has made less market impact than the others and because citalopram is a more recent entry into the market in most countries.

\section{Citalopram}

The low potential for drug interactions involving hepatic metabolism makes citalopram an SSRI to be considered in patients on other drugs for general

Table 3 Distinguishing features of individual selective serotonin reuptake inhibitors (SSRIs)

\section{SSRI Significant distinguishing features} (evidence level)

Citalopram No significant inhibition of hepatic enzymes $(\mathrm{P})$

Possibly less safe in overdose ${ }^{1}$ (III)

Fluoxetine Long elimination half-life (P) Inhibition of hepatic enzymes $(\mathrm{P})$ Slower onset of antidepressant action $^{1}$ (I)

Possibly causes more agitation and adverse dermatological reactions ${ }^{1}(\mathrm{I}-\mathrm{II})$

Abrupt treatment interruption least likely to cause discontinuation reactions ${ }^{1}(\mathrm{I})$

Probably safe in pregnancy and breast-feeding (III)

Associated with most stable pattern of prescription in primary care ${ }^{1}$ (II)

Fluvoxamine Inhibition of hepatic enzymes $(\mathrm{P})$ Less well tolerated, especially in higher doses ${ }^{1}$ (I)

More severe gastrointestinal sideeffects $^{1}$ (II)

Possibly less sexual dysfunction ${ }^{1}$ (I)

Paroxetine Inhibition of hepatic enzymes (P) Possibly causes more sedation and sexual dysfunction1 (II)

Possibly more weight gain during long-term use $^{1}(\mathrm{I})$

Abrupt treatment interruption most likely to cause discontinuation reactions ${ }^{1}$ (I-II)

Sertraline Little significant inhibition of hepatic enzymes $(\mathrm{P})$

Relatively safe in breast-feeding (III)

Levels of evidence: $\mathrm{P}$, evidence from pharmacological studies, usually of normal volunteers; I, from randomised controlled studies or meta-analysis; $\Pi$, from naturalistic or surveillance studies; III, from case reports. 1. Compared with other SSRIs. 
medical conditions or if consideration is given to adding an SSRI to other psychotropic medication. Vigilance is needed for possible drug interactions that may occur through other mechanisms and reference should be made to sources such as the British National Formulary (British Medical Association \& Royal Pharmaceutical Society, 2000). The concern about citalopram's safety in overdose must be kept in perspective, but the possibility of seizures or ECG changes occurring if more than $600 \mathrm{mg}$ (30 tablets) are taken suggests that caution should be exercised in its prescription for those at serious risk of overdose.

\section{Fluoxetine}

The long half-life of fluoxetine and norfluoxetine may underlie some of the differences between fluoxetine and other SSRIs. There is a disadvantage in prescribing this antidepressant if switching to a drug with the potential for serious interactions, such as a monoamine oxidase inhibitor (MAOI), is considered. If a switch is to be made a 5-week washout period is required. In practice, this is often not contemplated in advance but if it is, for example in treatment-resistant depression, another SSRI should be considered instead of fluoxetine.

The possible slower onset of antidepressant action of fluoxetine may be owing to a longer time taken to achieve therapeutic plasma concentrations. In situations where the speed of onset of therapeutic effect is particularly important, such as in severe depression, fluoxetine may not be the SSRI of choice. Patients in whom the long half-life may have advantages (and therefore for whom fluoxetine should be considered) include those who are poorly compliant and those in whom administration less frequent than daily is contemplated.

Fluoxetine's lower potential for discontinuation reactions is advantageous for patients who are prone to stop treatment suddenly or miss a few days' doses and for those who have suffered from troublesome SSRI discontinuation symptoms in the past.

The potential for drug interactions because of inhibition of cytochrome P450 enzymes indicates that fluoxetine should be used cautiously in patients on drugs for medical conditions or if consideration is given to combining an SSRI with other psychotropic medication.

Table 4 Guidelines for choosing an individual selective serotonin reuptake inhibitor (SSRI)

Situation

General

Previous good response or tolerability to a particular SSRI

Previous poor response or tolerabilityto a particular SSRI

Rapid antidepressant response particularly desirable

Concomitant medication or combination with TCA

Poor compliance with medication or likely to miss doses or interrupt treatment

Previous troublesome SSRI discontinuation reaction

High risk of self-poisoning limit amount of SSRI available

Previous troublesome sedation on an SSRI

Previous agitation on an SSRI

alternative class of antidepressant may be more appropriate (D)

Previous troublesome sexual

side-effects on an SSRI may be more appropriate (A)

Pregnancy

Breast-feeding

Likelihood of need to initiate or switch to drug with adverse interaction with SSRIs (e.g. MAOI)
Guidelines (level of recommendation)

Fluvoxamine not first-line owing to poorer tolerablity (A)

Choose the same SSRI unless other considerations (D)

Choose a different SSRI (D)

Consider any SSRI other than fluoxetine (B)

Consider citalopram or sertraline (C)

Consider fluoxetine, avoid paroxetine (A)

Avoid paroxetine, consider fluoxetine (A);

slowly taper SSRI (D)

Consider any SSRI other than citalopram (C);

Consider any SSRI other than paroxetine (C)

Consider any SSRI other than fluoxetine (C); an

Consider any SSRI other than paroxetine (B), in particular fluvoxamine (B); an alternative class of antidepressant

Consider fluoxetine (C)

Consider sertraline $(\mathrm{C})$

Avoid fluoxetine (C)

Recommendations based on the following levels of evidence (see Table 3): A, directly on level I; B, directly on level II or extrapolated from level I; C, directly on level III or P, or extrapolated from level I or II; and D, opinion. 
The possible increased potential for agitation and/or stimulatory side-effects is difficult to put in perspective, as many agitated or anxious patients tolerate fluoxetine without difficulty and, as with other drugs that have alleged stimulant effects, they may even obtain relief from tension and anxiety. However, if SSRI-induced agitation has previously occurred, then fluoxetine may not be the SSRI of choice.

There is a fairly large database of case reports of the use of fluoxetine in pregnancy and reports of over 20 cases of its use in breast-feeding with no evidence of an increased risk to the foetus or baby. Although the usual cautions about prescribing in these situations should be observed, fluoxetine appears to be an SSRI of choice if antidepressant treatment is deemed necessary during pregnancy. It is also probably safe during breast-feeding but its prolonged action puts it at a disadvantage compared with sertraline, on which there is a similar body of research data.

\section{Fluvoxamine}

More discontinuations occurred with fluvoxamine than other SSRIs in RCTs and this is consistent with the CSM and PEM findings of more troublesome gastrointestinal side-effects than those associated with other SSRIs. The observations suggest that fluvoxamine is not the best choice of SSRI in routine clinical practice. The potential for drug interactions involving cytochrome P-450 enzymes indicates that caution should be exercised when using fluvoxamine in patients receiving other drugs concurrently. There is the possibility that fluvoxamine causes less sexual dysfunction than other SSRIs and therefore it may be worth trying if patients have had troublesome sexual side-effects on other SSRIs.

\section{Paroxetine}

The potential for interactions involving hepatic metabolism indicates caution in using paroxetine with other drugs. The possible higher incidence of sedation and sexual side-effects calls for vigilance when paroxetine is prescribed for patients in whom these side-effects are especially undesirable or for those who have experienced the effects during previous treatment. The increased risk of discontinuation symptoms after sudden interruption of treatment with paroxetine suggests that it is not the SSRI of choice in patients who have previously experienced a troublesome discontinuation reaction and in those who are likely to miss doses or suddenly stop treatment.

\section{Sertraline}

The relatively low potential for drug interactions involving cytochrome P450 enzymes means that sertraline is an SSRI that should be considered in patients on concomitant medication. However, as with other SSRIs, vigilance for possible interactions that may occur through other mechanisms should be observed. There are reports in over 20 cases of the use of sertraline in mothers who are breast-feeding with no evidence of an increased risk to the baby. Although the usual caution concerning prescribing for lactating mothers needs to be observed, sertraline should be considered if SSRI treatment is necessary.

\section{References}

Anderson, I. M., Nutt, D. J. \& Deakin, J. F. W. (2000) Evidence-based guidelines for treating depressive disorders with antidepressants: a revision of the 1993 British Association for Psychopharmacology Guidelines. Journal of Psychopharmacology, 14, 3-20.

Arias, F., Padin, J. J., Gilaberte, I., et al (1998) Comparative efficacy and tolerability among different selective serotonin re-uptake inhibitors and venlafaxine in a naturalistic setting. International Journal of Psychiatry in Clinical Practice, 2, 255-260.

Borys, D. J., Setzer, S. C., Ling, L. J., et al (1992) Acute fluoxetine overdose: a report of 234 cases. American Journal of Emergency Medicine, 10, 115-120.

Boyer, P., Danion, J. M., Bisserbe, J. C., et al (1998) Clinical and economic comparison of sertraline and fluoxetine in the treatment of depression. A 6-month double-blind study in a primary-care setting in France. Pharmacoeconomics, 13, 157-169

British Medical Association \& Royal Pharmaceutical Society (2000) British National Formulary. London \& Wallingford: British Medical Journal \& Pharmaceutical Press.

Claxton, A. J., Chawla, A. J. \& Kennedy, S. (1999) Absenteeism among employees treated for depression. Journal of Occupational and Environmental Medicine, 41, 605611

- , Li, Z. \& McKendrick, J. (2000) Selective serotonin reuptake inhibitor treatment in the UK: risk of relapse or recurrence of depression. British Journal of Psychiatry, 177, $163-168$.

Dechant, K. L. \& Clissold S. P. (1991) Paroxetine. A review of its pharmacodynamic and pharmacokinetic properties, and therapeutic potential in depressive illness. Drugs, 41, 225-253.

Edwards, J. G. \& Anderson, I. (1999) Systematic review and guide to selection of selective serotonin reuptake inhibitors. Drugs, 57, 507-533.

-, Inman, W. H. W., Wilton L., et al (1997) Drug safety monitoring of 12692 patients treated with fluoxetine. Human Psychopharmacology, 12, 127-137.

Fava, M., Rosenbaum, J., Judge, R., et al (1998) Fluoxetine versus sertraline and paroxetine in major depression: longterm changes in weight. European Neuropsychopharmacology, 8 (Suppl. 2), S204.

Glassman, A. H. (1997) Citalopram toxicity. Lancet, 350, 818.

Goldstein, D. J. \& Sundell, K. (1999) A review of the safety of selective serotonin reuptake inhibitors during pregnancy. Human Psychopharmacology, 14, 319-324.

Goodnick, P. J. \& Goldstein, B. J. (1998a) Selective serotonin reuptake inhibitors in affective disorders. I. Basic pharmacology. Journal of Psychopharmacology, 12, S5-S20. 
— \& - (1998b) Selective serotonin reuptake inhibitors in affective disorders. II. Efficacy and quality of life. Journal of Psychopharmacology, 12, S21-S54.

Gregor, K. J., Overhage, J. M., Coons, S. J., et al (1994) Selective serotonin reuptake inhibitor dose titration in the naturalistic setting. Clinical Therapeutics, 16, 306-315.

Hamilton, M. (1960) A rating scale for depression. Journal of Neurology, Neurosurgery and Psychiatry, 23, 56-62.

Henry, J. A. (1991) Overdose and safety with fluvoxamine. International Clinical Psychopharmacology, 6 (Suppl. 3), 4145

- Alexander, C. A. \& Sener, E. K. (1995) Relative mortality from overdose of antidepressants. British Medical Journal, 310, 221-224

Hylan, T. R., Dossenbach, M., Meneades, L., et al (1998) Antidepressant use in the psychiatrist setting in Austria: a comparison of citalopram, fluoxetine, and paroxetine. Journal of Serotonin Research, 4, 295-303.

---, Meneades, L., Crown, W. H., et al (1999) SSRI antidepressant use patterns and their relation to clinical global impression scores: a naturalistic study. Journal of Affective Disorders, 52, 111-119.

Isacsson, G. \& Bergman, U. (1996) Risks with citalopram in perspective. Lancet, 348, 1033.

Klein-Schwartz, W. \& Anderson B. (1996) Analysis of sertraline-only overdoses. American Journal of Emergency Medicine, 14, 456-458.

Lawrenson, R. A., Tyrer, F., Newson, R. B., et al (2000) The treatment of depression in UK general practice: selective serotonin reuptake inhibitors and tricyclic antidepressants compared. Journal of Affective Disorders, 59, 149-157.

Mackay, F. J., Dunn, N. R., Wilton, L.V., et al (1997) A comparison if fluvoxamine, fluoxetine, sertraline and paroxetine examined by observational cohort studies. Pharmacoepidemiology and Drug Safety, 6, 235-246.

---, Dunn, N. R., Martin, R. M., et al (1999). Newer antidepressants: a comparison of tolerability in general practice. British Journal of General Practice, 48, 892-896.

Michelson, D., Fava, M., Amsterdam, J., et al (2000) Interruption of selective serotonin reuptake inhibitor treatment. Double-blind, placebo-controlled trial. British Journal of Psychiatry, 176, 363-368.

Montejo-Gonzalez, A. L., Llorca, G., Izquierdo, J. A., et al (1997) SSRI-induced sexual dysfunction: fluoxetine, paroxetine, sertraline, and fluvoxamine in a prospective, multicenter, and descriptive clinical study of 344 patients. Journal of Sexual and Marital Therapy, 23, 176-194.

Nemeroff, C. B., Ninan, P. T., Ballenger, J., et al (1995) Doubleblind multicenter comparison of fluvoxamine verus sertraline in the treatment of depressed outpatients. Depression, 3, 163-169.

Öström, M., Eriksson, A., Thorson, J., et al (1996) Fatal overdose with citalopram. Lancet, 348, 339-340.

Personne, M., Sjoberg, G. \& Persson, H. (1997) Citalopram overdose - review of cases treated in Swedish hospitals. Journal of Toxicology and Clinical Toxicology, 35, 237-240.

Rosenbaum, J. F., Fava, M., Hoog, S. L., et al (1998) Selective serotonin reuptake inhibitor discontinuation syndrome: a randomized clinical trial. Biological Psychiatry, 44, 77-87.

Russell, J. M., Berndt, E. R., Miceli, R., et al (1999) Course and cost of treatment for depression with fluoxetine, paroxetine, and sertraline. American Journal of Managed Care, 5, 597-606.

Sclar, D. A., Robison, L. M., Skaer, T. L., et al (1995) Antidepressant pharmacotherapy: economic evaluation of fluoxetine, paroxetine and sertraline in a health maintenance organization. Journal of International Medical Research, 23, 395-412.

_-, Skaer, T. L., Robison, L. M., et al (1999) Economic appraisal of citalopram in the management of singleepisode depression. Journal of Clinical Psychopharmacology, 19, 47S-54S

Teicher, M. H., Glod, C. \& Cole, J. O. (1990) Emergence of intense suicidal preoccupation during fluoxetine treatment. American Journal of Psychiatry, 147, 207-210.
Waldinger, M. D., Hengeveld, M. W., Zwinderman, A. H., et al (1998) Effect of SSRI antidepressants on ejaculation: a double-blind, randomized, placebo-controlled study with fluoxetine, fluvoxamine, paroxetine, and sertraline. Journal of Clinical Psychopharmacology, 18, 274-281.

Wilton, L. V., Pearce, G. L., Martin, R. M., et al (1998) The outcomes of pregnancy in women exposed to newly marketed drugs in general practice in England. British Journal of Obstetrics and Gynaecology, 105, 882-889.

Yoshida, K., Smith, B. \& Kumar, R. C. (1999) Psychotropic drugs in mother's milk: a comprehensive review of assay methods, pharmacokinetics and of safety in breastfeeding. Journal of Psychopharmacology, 13, 64-80.

\section{Multiple choice questions}

1. The following are true of the quality of evidence available from the specified type of study:

a randomised controlled studies provide evidence of efficacy rather than effectiveness

b case studies allow comparison of the relative frequency of adverse events for different SSRIs

c PEM controls for bias in allocating particular types of patient to individual SSRIs

$\mathrm{d}$ post-marketing surveillance is vulnerable to biases arising from selective reporting of adverse events

e naturalistic studies cannot include randomisation to different treatment arms.

2. Pharmacological differences that appear to be important in choosing between SSRIs include:

a elimination half-life

b affinity for sigma opioid receptors

c potency in inhibiting 5-HT reuptake

d selectivity in inhibiting 5-HT reuptake

e inhibition of hepatic cytochrome P450 isoenzymes.

3. Randomised controlled trials in which SSRIs have been compared with each other suggest that: a fluoxetine may have a slower onset of antidepressant action than other SSRIs

b fluvoxamine is less well tolerated than other SSRIs

c sertaline is less anxiolytic than other SSRIs

$\mathrm{d}$ paroxetine is more likely to cause discontinuation symptoms than other SSRIs

e citalopram is more anxiolytic than other SSRIs.

4. The following differences between SSRIs are suggested by surveillance, monitoring or case report data:

a citalopram may have more potential to cause serious adverse events in overdose than other SSRIs 
b paroxetine and fluovoxamine are associated with more sedation than other SSRIs

c fluoxetine is less often associated with dose escalation in primary care than other SSRIs

d fluvoxamine is associated with more severe gastrointestinal side-effects than other SSRIs

e sertraline is associated with more sexual dysfunction than other SSRIs.

5. The following statements are true:

a switching from an SSRI to an MAOI carries no risks

b citalopram has less effect on P450 enzymes than fluoxetine

c sertraline is more potentially lethal in overdose than other SSRIs d treatment with fluoxetine during pregnancy increases the risk of spontaneous abortion

e there is a sparcity of data on the effects of SSRIs during breast-feeding.

\begin{tabular}{llllllllll}
\multicolumn{2}{l}{ MCQ answers } & & & & & & \\
1 & & 2 & & 3 & & 4 & & 5 & \\
a & T & a & T & a & T & a & T & a & F \\
b & F & b & F & b & T & b & T & b & T \\
c & F & c & F & c & F & c & T & c & F \\
d & T & d & F & d & T & d & T & d & F \\
e & F & e & T & e & F & e & F & e & T
\end{tabular}

\section{The Royal College of Psychiatrists Conferences in 2001}

Annual Meeting

9-13 July 2001

QEII Centre, London

Joint Meeting of Philosophy and WPA Section

14 July 2001

SOAS, London

Annual Residential Meeting of the Child and Adolescent Psychiatry Faculty

3-5 October 2001

University of York

Faculty for the Psychiatry of Learning Disability Residential Meeting

10-12 October 2001

Jarvis Hotel, Chester

Section of Rehabilitation and Social Psychiatry Residential Meeting

15-16 November 2001

Gosforth Swallow, Newcastle

Fitness to Drive (Old Age Psychiatry)

5 December 2001

$\mathrm{SCI}$, London

For further information please contact:

Conference Office, Royal College of Psychiatrists, 17 Belgrave Square, London, SW1X 8PG.

Tel: +44 (0)2072352351 Ext 142 Fax: +44 (0)2072451231 Website: www.rcpsych.ac.uk 